\title{
Front Matter: Volume 11028
}

, "Front Matter: Volume 11028," Proc. SPIE 11028, Optical Sensors 2019, 1102801 (16 July 2019); doi: 10.1117/12.2535406

SPIE. Event: SPIE Optics + Optoelectronics, 2019, Prague, Czech Republic 


\title{
PROCEEDINGS OF SPIE
}

\section{Optical Sensors 2019}

\author{
Francesco Baldini \\ Jiri Homola \\ Robert A. Lieberman \\ Editors
}

\section{1-4 April 2019}

Prague, Czech Republic

Sponsored by

SPIE

Cooperating Organisations

ELI Beamlines (Czech Republic)

Laserlab Europe

European Optical Society

HiLASE (Czech Republic)

Published by

SPIE 
The papers in this volume were part of the technical conference cited on the cover and title page. Papers were selected and subject to review by the editors and conference program committee. Some conference presentations may not be available for publication. Additional papers and presentation recordings may be available online in the SPIE Digital Library at SPIEDigitalLibrary.org.

The papers reflect the work and thoughts of the authors and are published herein as submitted. The publisher is not responsible for the validity of the information or for any outcomes resulting from reliance thereon.

Please use the following format to cite material from these proceedings:

Author(s), "Title of Paper," in Optical Sensors 2019, edited by Francesco Baldini, Jiri Homola, Robert A. Lieberman, Proceedings of SPIE Vol. 11028 (SPIE, Bellingham, WA, 2019) Seven-digit Article CID Number.

ISSN: 0277-786X

ISSN: 1996-756X (electronic)

ISBN: 9781510627222

ISBN: 9781510627239 (electronic)

Published by

SPIE

P.O. Box 10, Bellingham, Washington 98227-0010 USA

Telephone +1 3606763290 (Pacific Time) · Fax +1 3606471445

SPIE.org

Copyright @ 2019, Society of Photo-Optical Instrumentation Engineers.

Copying of material in this book for internal or personal use, or for the internal or personal use of specific clients, beyond the fair use provisions granted by the U.S. Copyright Law is authorized by SPIE subject to payment of copying fees. The Transactional Reporting Service base fee for this volume is $\$ 18.00$ per article (or portion thereof), which should be paid directly to the Copyright Clearance Center (CCC), 222 Rosewood Drive, Danvers, MA 01923. Payment may also be made electronically through CCC Online at copyright.com. Other copying for republication, resale, advertising or promotion, or any form of systematic or multiple reproduction of any material in this book is prohibited except with permission in writing from the publisher. The CCC fee code is 0277$786 \times / 19 / \$ 18.00$.

Printed in the United States of America by Curran Associates, Inc., under license from SPIE.

Publication of record for individual papers is online in the SPIE Digital Library.

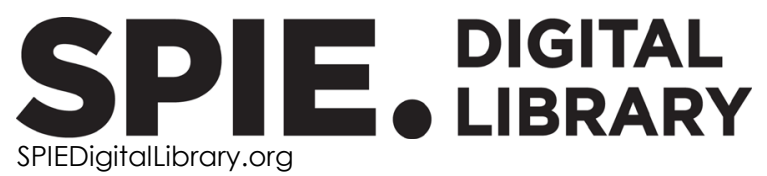

Paper Numbering: Proceedings of SPIE follow an e-First publication model. A unique citation identifier (CID) number is assigned to each article at the time of publication. Utilization of CIDs allows articles to be fully citable as soon as they are published online, and connects the same identifier to all online and print versions of the publication. SPIE uses a seven-digit CID article numbering system structured as follows:

- The first five digits correspond to the SPIE volume number.

- The last two digits indicate publication order within the volume using a Base 36 numbering system employing both numerals and letters. These two-number sets start with 00, 01, 02, 03, 04, 05, 06, 07, 08, 09, OA, OB ... 0Z, followed by 10-1Z, 20-2Z, etc. The CID Number appears on each page of the manuscript. 


\title{
Contents
}

\author{
ix Authors \\ xiii Conference Committee
}

SESSION $1 \quad$ COMPONENTS AND DATA PROCESSING METHODS

1102803 A solid carbon source based high performance mono/bi layer graphene/SiNWs heterojunction NIR photodetector [11028-2]

$1102804 \quad$ Laser scanning module with large sending aperture and inherent high angular position accuracy for 3D LiDAR [11028-3]

1102805 Optical choppers with spherical shafts: an optomechanical analysis [11028-4]

\section{SESSION 2 FIBER OPTIC SENSORS I}

1102806 Design challenges of a birefringent FBG optical sensing system [1 1028-6]

1102808 Comparison of solution approaches for distributed humidity sensing in perfluorinated gradedindex polymer optical fibers [1 1028-8]

11028 OA Fiber-optic sensor for detecting electric current pulses [11028-10]

SESSION 3 FIBER OPTIC SENSORS II

11028 OC Multi-application miniature fiber optic interferometer [1 1028-14]

11028 OD Random matrix theory based distributed acoustic sensing [1 1028-15]

\section{SESSION 4 NOVEL APPROACHES IN OPTICAL SENSING I}

11028 OG Do you need a tunable laser for resonant cavity optical sensors? [1 1028-18]

$11028 \mathrm{OH} \quad$ High numerical aperture waveguide absorption filter for fluorescence detection [1 1028-19]

11028 Ol Enhancing light-matter interaction in all-dielectric photonic crystal metasurfaces [1 1028-20] 
SESSION $5 \quad$ NOVEL APPROACHES IN OPTICAL SENSING II

$11028 \mathrm{OL} \quad$ Advances in random lasing sensing [11028-23]

SESSION 6 OPTICAL BIOSENSORS

11028 OR Smartphone biosensor for Salmonella and Amitriptyline [1 1028-29]

SESSION 7 PLASMONIC SENSING

$11028 \mathrm{OW} \quad$ Surface plasmon resonance imaging for detection of drug metabolites in water [11028-31]

11028 OX The main ways of applying nanostructured metasurfaces in rotation angle sensors [1 1028-65]

\section{SESSION 8 CHEMICAL SENSORS}

$1102810 \quad$ NIR transillumination system for in vivo functional imaging [1 1028-38]

\section{POSTER SESSION}

1102812 An improved dense dark vegetation based algorithm for aerosol optical thickness retrieval from hyperspectral data [1 1028-5]

1102813 Functional near infrared spectroscopy (fNIRS) in pigmented subjects: a maneuver to confirm sufficient transcutaneous photon transmission for measurement of hemodynamic change in the anterior cortex [11028-41]

1102815 Emissivity-free thermometer using hyperspectral camera and multivariate analysis and its application to steel manufacturing processes [1 1028-45]

1102816 OPTICAL sensors in IOT [11028-46]

$1102818 \quad$ Estimation of pore sizes using laser absorption in molecular oxygen gas enclosed in mesoporous alumina [1 1028-48]

11028 1A Impact of surface plasmon resonance on angular spin splitting of light [1 1028-50]

11028 1B High spatial resolution physical and chemical sensing based on BOFDA [1 1028-51]

11028 1C Wide dynamic range laser remote sensing detection chain [1 1028-52]

iv 
$11028 \mathrm{lF} \quad$ Smart Home room's occupancy monitoring using Fiber Bragg grating sensor [1 1028-55]

$1102811 \quad$ Fiber optic Raman distributed temperature sensor based on an ultrashort pulse mode-locked fiber laser [1 1028-59]

$11028 \mathrm{lJ} \quad$ Spectral response filtering by lateral scanning of Silicon NSOM photodetector with subwavelength aperture [1 1028-60]

$110281 \mathrm{~K} \quad$ Humidity sensor based on optical fiber coated with agarose gel [11028-61]

11028 IL Golden layer characterization using a phase shift induced by surface plasmon resonance [11028-62]

$110281 \mathrm{M} \quad$ Noncontact optical distance and speed sensor using novel offsetless spatial frequency filters [11028-63]

$110281 \mathrm{~N}$ Effect of the misalignments of ring confocal resonator on its optical properties [1 1028-64]

1102810 Optical readout of acoustic responses of an external-cavity diode laser [1 1028-66]

$110281 \mathrm{P} \quad$ Surface electromagnetic wave sensor utilizing a one-dimensional photonic crystal [1 1028-67]

$110281 Q$ Investigation of parameters of the Bessel beam formed by an axicon [1 1028-68]

11028 is Optical fibre fuse effect based sensor for magnetic field monitoring [1 1028-70]

11028 IT Highly sensitive carboxyl-graphene oxide-based SPR immunosensor for the detection of CA199 biomarker [11028-71]

$110281 \mathrm{U}$ Metal coated fiber sensor for laser power measurements with enhanced sensitivity [1 1028-72]

1102820 Low-cost intrinsic optical fiber FPI sensor for knee kinematic gait analysis and e-Health architecture [11028-79]

$1102822 \quad$ Hemoglobin detection using a graphene oxide functionalized side-polished fiber sensor [11028-82]

1102823 Plasmonic biosensor detected human chorionic gonadotropin with naked eye [11028-83]

$1102828 \quad$ Fiber Bragg Gratings strain sensors for deflection estimation of a two-dimensional structure [11028-88]

1102829 Semi-automated method for views integration based on alternative color and geometry characteristics [1 1028-89]

$110282 \mathrm{~B} \quad$ Optical fiber based heterodyne interferometry for non-invasive and non-contact oil viscosity measurement [1 1028-91] 
11028 2C Matrix of piezoelectric resonators for registration of spatial distribution of laser radiation [11028-92]

$110282 \mathrm{E} \quad$ Fabrication and characterization of long period gratings in pure-silica fibers [1 1028-94]

$110282 \mathrm{~F}$ Metallic-Dielectric colloidal photonic crystal on the multimode optical fiber tip: preliminary results as optical fiber SERS probe [11028-95]

$110282 \mathrm{G} \quad$ Low cost flexible $1.1 \mu \mathrm{m}-1.6 \mu \mathrm{m}$ photodetector fabricated by hydrothermal grown large area MoSe2 nanostructures [1 1028-97]

$110282 \mathrm{H} \quad$ SPR-based fiber optic sensor in NIR region [1 1028-98]

$1102821 \quad$ Spatial selectivity and sensitivity measurement of optoelectronic devices by scanning microscopy [1 1028-99]

11028 2K Perimetric monitoring: A comparison of a classical seismic sensor and fiber-optic interferometric sensor [1 1028-101]

$110282 \mathrm{~L} \quad$ Adsorption kinetics of proteins to biomaterials measured by reflectometric interference spectroscopy [11028-102]

$110282 \mathrm{M} \quad$ Bioinspired optical fiber sensor for simultaneous shear and vertical forces monitoring [1 1028-103]

1102820 Coating of modified plastic optical fibers with proteins for chemical sensing and biosensing: preliminary studies [1 1028-105]

11028 2P Interferometric scattering (iSCAT) microscopy with optimized reference wave [1 1028-106]

$110282 Q \quad$ Methods of signal averaging for a multimode fiber interferometer: an experimental study [11028-107]

$110282 S \quad$ Noise characteristics and localisation precision in ultrafast interferometric scattering (iSCAT) microscopy [1 1028-109]

$110282 T \quad$ Tm-doped fiber laser with control of spectral dynamics [1 1028-1 10]

$110282 \mathrm{X}$ Design and simulation of image nonlinear processing relational preprocessor based on iterational sorting node [1 1028-115]

$110282 Y \quad$ Improvements of the PLD (Pulsed Laser Deposition) method for fabricating photocathodes in ICMOS (Intensified CMOS) sensors [1 1028-116]

$110282 Z \quad H y d r o g e n$ sensors based on plasmonic nanostructures present on palladium films [11028-117]

1102830 Modelling and fabrication of novel SERS sensor chips for chemical and biological sensing applications [11028-118]

1102831 Optical sensors based on palladium and polymer-coated optical fiber resonators [1 1028-119] 
1102833 Land surface temperature and emissivity retrieval from time-series thermal infrared data [11028-123]

1102836 FDTD modelling and simulation of organic photo detector using photonic crystals [1 1028-126]

1102837 Design of encapsulation of fiber Bragg grating for the traffic applications [1 1028-127]

1102838 Growth and characterization of calcium-doped cesium iodide (Csl:Ca) optical crystals for radiation detection [1 1028-128]

1102839 Evaluation of cortical neuroexcitation in urinary urgency using simultaneous near infrared spectroscopy of the brain and bladder with quantification of sensation [1 1028-129] 
Proc. of SPIE Vol. 11028 1102801-8

\section{Downloaded From: https://www.spiedigitallibrary.org/conference-proceedings-of-spie on 26 Apr 2023
Terms of Use: https://www.spiedigitallibrary.org/terms-of-use}




\section{Authors}

Numbers in the index correspond to the last two digits of the seven-digit citation identifier (CID) article numbering system used in Proceedings of SPIE. The first five digits reflect the volume number. Base 36 numbering is employed for the last two digits and indicates the order of articles within the volume. Numbers start with 00, 01, 02, 03, 04, 05, 06, 07, 08, 09, OA, OB...0Z, followed by 10-12, 20-2Z, etc.

Adinolfi, Barbara, $\mathrm{OH}$

Agarwal, Neha, 03

Al-Basheer, Watheq, 18

Alberto, Nélia J., 1S, 20, 2M

Aljalal, Abdulaziz, 18

Al-Saudi, Ahmed, 18

Alviggi, Mariagrazia, 28

Amaral, Vitor, $1 \mathrm{~S}$

André, Paulo, 1S, 20, 2M

Antunes, Paulo, 1S, 20, 2M

Arrizabalaga, Oskar, OC

B.M., Chaya, 36

Baldini, Francesco, $\mathrm{OH}$

Bang, Seoung-Chul, $2 Y$

Bavili, N., 31

Bazarov, Timur O., 2C

Bello, Valentina, 10

Belo, João H., is

Berneschi, Simone, $\mathrm{OH}$

Bernini, Romeo, $\mathrm{OH}, 1 \mathrm{~B}$

Beuth, Thorsten, 2l

Bierl, Rudolf, OW

Bilro, L., 20

Bisyarin, Michail, 2Q

Bodo, Elisabetta, 10

Bogatscher, Siegwart, 04

Bozhko, Dmitry, $2 Q$

Bronkhorst, Mathijs, 13

Budarnykh, A. E., 2T

Bujak, L., 2S

Bunsch, Eryk, 29

Cabrini, Stefano, 01

Çabuk, Sercan, 2

Campopiano, Stefania, 28, 2E, 2F

Canale, Vincenzo, 28

Catalano, Ester, 1B

Catalano, Rachele, 10

Cavalieri, Stefano, $0 \mathrm{~L}$

Chaliyawala, Harsh A., 03

Chapalo, Ivan, $2 Q$

Chernutsky, Anton O., 11

Chiavaioli, Francesco, $\mathrm{OH}$

Chiu, Nan-Fu, 1T, 23

Chlebus, R., $1 \mathrm{~L}$

Choi, Hyeunseok, $2 Y$

Choi, Ji Yeon, 2B

Čičala, M., $2 S$

Ciprian, D., 1L, 1P

Colier, Willy N. J. M., 13
Coscetta, Agnese, 1B

Csukas, Eduard-Sebastian, 05

Danenkov, lliya S., 16

Das, Samaresh, $2 \mathrm{G}$

De Luca, Anna Chiara, 01

Della Pietra, Massimo, 28

Dhawan, Anuj, 2Z, 30

Dhyani, Veerendra, $2 \mathrm{G}$

Di Palma, Pasquale, 28, 2F

Dietzel, A., OR

Domingues, Maria Fátima, 1S, 20, 2M

Duma, Virgil-Florin, 05

Dvoretskiy, Dmitriy A., 11

Efremova, Ekaterina A., OX

Eom, Jonghyun, 2B

Eom, Joo Beom, 2B

Eryürek, M., 31

Esposito, Flavio, 2E

Fajkus, Marcel, 1F, 37

Fan, Shi-Yuan, $1 T$

Farnan, Martin, 06

Fedorov, Vladimir V., 2C

Ferreira, Marta S., $1 \mathrm{~K}$

Filatov, Yuri V., IN

Fini, Lorenzo, OL

Fischer, Johannes, OW

Foryś, Piotr, 29

Frey, Jochen, $1 \mathrm{M}$

Gamal, Rania, 0G

Gao, Caixia, 12, 33

Gasmi, Khaled, 18, 1C

Gauglitz, Günter, OR, 2L

Ghosh, Rajib R., 2Z, 30

Giannetti, Ambra, $\mathrm{OH}$

Giordano, Michele, 2F

Gorelaya, Alina V., 1N

Gryga, M., 1P

Gubin, Vladimir P., OA

Gupta, Govind, 03

Hasselbach, Jürgen, 04

Hatano, N., 15

Hausler, Peter, OW

Hecht, L., OR

Heckscher, Simon, OW

Hirota, S., 15

Hirsch, Thomas, ow

Hlubina, P., 1L, 1P

Holanova, Kristyna, 2P

Hossain, Mosaddek, 21 
Hutterer, Johanna, OR, 2L ladicicco, Agostino, 28, 2E, 2F Ibrahim, Selwan K., 06 Ignesti, Emilio, OL Iureva, Radda A., 16 Jaiswal, Mangesh, 2Z, 30 Jalowiczor, Jakub, 2K Janoschek, Peter, $2 \mathrm{~L}$ Jaros, Rene, 2K, 37 Jurdova, Dominika, 2P K., Narayan, 36 Kablukov, S. I., 2T Kang, Sung Bok, $2 Y$ Kang, Yong-Woo, $2 Y$ Kaňok, R., $1 \mathrm{~L}$

Karasik, Valeriy E., 11 Karelits, Matityahu, $1 \mathrm{~J}$ Karsenty, Avi, $1 \mathrm{~J}$ Kaur, Baljinder, 2H Kemmochi, M., 15 Khramov, Ivan, $1 \mathrm{U}$ Kim, Daegil, 22

Kim, Yang-Sook, 2 Y

Kiraz, A., 31

Kirk, Andrew, OG

Klausner, Adam, 39 Konyashkin, Alexey V., 2C Korolkov, Andrey E., 2C Kotov, Oleg, $2 Q$ Kovalev, Michael S., 1Q Krasilenko, Vladimir G., 2X Krasin, George K., 1Q Krebber, Katerina, 08 Kukaev, Alexander S., $1 \mathrm{~N}$ Kulsum, Umme, 36 Kumari, Preeti, 2G

Kun, Li, 33 Lang, Yaopu, $1 \mathrm{~A}$ Lara Yépez, Sofía Natalí, OI Lazarev, Alexander A., $2 X$ Lech, Krzysztof, 29 Lee, Dae-Hee, $2 Y$

Li, Chuanrong, 12, 33

Liehr, Sascha, 08

Liu, Qinggang, 1A

Liu, Yaokai, 12, 33

Lobach, I. A., 2T

Ma, Lingling, 12

Macnab, Andrew J., 13, 39

Maity, Sarmistha, $2 \mathrm{G}$

Malyshev, Alexander, $2 Y$

Markiewicz, Łukasz, 29

Marques, Carlos, is

Martelli, Fabrizio, OL

Martinek, Radek, IF

McCue, Raymond, 06

Mec, Pavel, 37

Merlo, Sabina, 10

Michoński, Jakub, 29

Minardo, A., 1B
Mocella, Vito, Ol

Moon, Bongkon, $2 Y$

Morova, B., 31

Morshnev, Sergey K., OA

Mukhankov, Denis M., 2C

Mukhopadhyay, Indrajit, 03

Mukisa, Ronald, 13

Musto, Pellegrino, 2F

Najih, Mohamed, OG

Nepomuceno, Ana, 20

Nickel, Malte, $1 M$

Nikitovich, Diana V., 2X

Nikolaeva, Natalia A., OX

Nogueira, R., 20

Nosov, Pavel A., 1Q

Novais, Susana, $1 \mathrm{~K}$

Novak, Martin, 2K, 37

Nuntawong, Noppadon, 38

O'Dowd, John A., 06

Ölçer, ibrahim, OD

Öncü, Ahmet, OD

Orekhov, llya O., 11

Oshige, T., 15

Ososkov, Yan Zh., 11

Palumbo, Giovanna, 28

Pannico, Marianna, 2F

Park, Anjin, 2B

Park, Won-Kee, $2 Y$

Park, Youngsik, $2 Y$

Penzo, Erika, Ol

Persichetti, Gianluca, $\mathrm{OH}$

Petrov, Alexandr, 2Q

Pfab, Christina, OW

Piliarik, Marek, 2P, $2 S$

Pinka, Miroslav, 2K

Pinto, João L., $1 \mathrm{~K}$

Pizzurro, Sara, 10

Pniov, Alexey B., 11

Przhiyalkovskiy, Yan V., OA

Purohit, Zeel, 03

Qari, Samer, 18

Qian, Yonggang, 12, 33

Qin, Lin, 10

Qin, Zirui, 1A

Qiu, Shi, 12, 33

Qiu, Yuanyuan, 33

Radwan, Ayman, 20

Ramos, António, 2M

Ray, Abhijit, 03

Rembe, Christian, 04

Ren, Huan, 10

Rendina, Ivo, 0 I

Ricklefs, Ubbo, $1 \mathrm{M}$

Rocon, Eduardo, 2M

Romano, Silvia, 0

Rossi Borghesano, Marco, 10

Ryabushkin, Oleg A., 1U, 2C

Saengkaew, Phannee, 38

Sáez de Ocáriz, Idurre, OC

Sampath, Umesh, 22 
Sansone, Lucia, $2 \mathrm{~F}$

Santos-Silva, T., 20

Savichev, llya A., 2C

Sazonkin, Stanislav G., 11

Sazonov, Aleksandr I., OA

Schenk, F., OR

Schreier, Andy, 08

Sequeira, F., 20

Shaidullin, Renat, $1 \mathrm{U}$

Shalymov, Egor V., OX, IN

Sharma, Anuj K., 2H

Sharma, Yashna, 2Z, 30

Shen, Qingfeng, 33

Shoev, Vladislav, OX

Sill, A., OR

Silva, Hugo, 2M

Sitnik, Robert, 29

Skvortsov, M. I., 2T

Song, Minho, 22

Speich, John, 39

Srivastava, Anubhav, 2E

Starostin, Nikolay I., OA

Stolarik, Martin, IF, 2K

Stothers, Lynn, 13, 39

Tang, Lingli, 12, 33

Tavares, Cátia, 20, 2M

Testa, Genni, $\mathrm{OH}$

Thanachayanont, Chanchana, 38

Tiemerding, T., OR

Timko, Andrei S., 16

Tombelli, Sara, $\mathrm{OH}$

Tommasi, Federico, OL

Trono, Cosimo, $\mathrm{OH}$

Vala, Milan, 2P, 2S

Vanus, Jan, IF

Vasilkov, Sergey D., 16

Vedenkin, Nikolay, $2 Y$

Venediktov, Vladimir Yu., OX, IN

Venediktova, Anastasia $\vee$., OX

Villatoro, Joel, OC

Vinogradov, Maxim A., 1Q

Vladimirskaya, A. D., 2T

Vlasov, Sergey M., 16

Wadeng, Imron, 38

Wang, Junmin, 10

Wang, Ning, 12, 33

Wang, Yanhua, 10

Wasinski, Frank, $1 \mathrm{M}$

Weber, P., OR

Witas, Karel, 2K

Wolf, A. A., 2T

Wosniok, Aleksander, 08

Wu, Hua, 33

Wunderlich, Lukas, OW

Yen, Yu-Chieh, 23

Yilgor, E., 31

Yilgor, I., 31

Yordsri, Visittapong, 38

Yue, Chong, 1A

Zabka, Stanislav, 1F, 2K, 37
Zeni, Luigi, 1B

Zhou, Xinglin, 1A

Zito, Gianluigi, 0 I

Zotov, Kirill V., 2C

Zubia, Joseba, OC

Zywietz, Urs, 2I 
Proc. of SPIE Vol. 11028 1102801-12

Downloaded From: https://www.spiedigitallibrary.org/conference-proceedings-of-spie on 26 Apr 2023 Terms of Use: https://www.spiedigitallibrary.org/terms-of-use 


\title{
Conference Committee
}

\author{
Symposium Chairs
}

Bedrich Rus, ELI Beamlines, Institute of Physics of the CAS, v.v.i. (Czech Republic)

Chris Edwards, STFC Rutherford Appleton Laboratory

(United Kingdom)

Saša Bajt, Deutsches Elektronen-Synchrotron (Germany)

Ivo Rendina, Istituto per la Microelettronica e Microsistemi (Italy)

Honorary Symposium Chair

Erich Spitz, French Academy of Sciences, National Academy of Technologies (France), Advisor to Thales (France)

Conference Chairs

Francesco Baldini, Istituto di Fisica Applicata Nello Carrara (Italy) Jiri Homola, Institute of Photonics and Electronics of the ASCR, v.v.i. (Czech Republic)

Robert A. Lieberman, Lumoptix, LLC (United States)

Conference Programme Committee

Loïc J. Blum, Université Claude Bernard Lyon 1 (France)

Eduard Brynda, Institute of Macromolecular Chemistry of the ASCR, v.v.i. (Czech Republic)

Stefania Campopiano, Università degli Studi di Napoli Parthenope (Italy)

Artur Dybko, Warsaw University of Technology (Poland)

Günter G. Gauglitz, Eberhard Karls Universität Tübingen (Germany)

Pedro Jorge, INESC Porto (Portugal)

Aleksandra Lobnik, University of Maribor (Slovenia)

Ramaier Narayanaswamy, The University of Manchester (United Kingdom)

Terro Soukka, University of Turku (Finland)

Reinhardt Willsch, Institut für Photonische Technologien e.V. (Germany) 


\section{Session Chairs}

1 Components and Data Processing Methods

Romeo Bernini, Istituto per il Rilevamento Elettromagnetico dell'Ambiente (Italy)

2 Fiber Optic Sensors I

Francesco Baldini, Istituto di Fisica Applicata "Nello Carrara" (Italy)

3 Fiber Optic Sensors II

Francesco Baldini, Istituto di Fisica Applicata "Nello Carrara" (Italy)

4 Novel Approaches in Optical Sensing I

Günter Gauglitz, Eberhard Karls Universität Tübingen (Germany)

5 Novel Approaches in Optical Sensing II

Andrew G. Kirk, McGill University (Canada)

6 Optical Biosensors

Niko Hildebrandt, Centre de Nanosciences et de Nanotechnologies (France)

$7 \quad$ Plasmonic Sensing

Jirí Homola, Institute of Photonics and Electronics of the CAS, v.v.i. (Czech Republic)

8 Chemical Sensors

Nongjian Tao, Arizona State University (United States) 